\title{
Lumen
}

Selected Proceedings from the Canadian Society for Eighteenth-Century Studies

\section{Savoirs et Saveurs}

\section{Beatrice Fink}

Volume 19, 2000

Material Productions \& Cultural Construction

Culture matérielle \& Constructions discursives

URI : https://id.erudit.org/iderudit/1012314ar

DOI : https://doi.org/10.7202/1012314ar

Aller au sommaire du numéro

Éditeur(s)

Canadian Society for Eighteenth-Century Studies / Société canadienne d'étude du dix-huitième siècle

\section{ISSN}

1209-3696 (imprimé)

1927-8284 (numérique)

Découvrir la revue

Citer cet article

Fink, B. (2000). Savoirs et Saveurs. Lumen, 19, 39-53.

https://doi.org/10.7202/1012314ar

Copyright $@$ Canadian Society for Eighteenth-Century Studies / Société canadienne d'étude du dix-huitième siècle, 2000
Ce document est protégé par la loi sur le droit d'auteur. L'utilisation des services d'Érudit (y compris la reproduction) est assujettie à sa politique d'utilisation que vous pouvez consulter en ligne.

https://apropos.erudit.org/fr/usagers/politique-dutilisation/ 


\section{Savoirs et Saveurs}

Trop souvent méconnu ou déconsidéré, le discours culinaire des Lumières, plus exactement son expression française, mérite pourtant notre attention à plus d'un titre. Il la mérite en premier lieu à cause de certains écrits qu'il s'agit de valoriser dans la mesure où ils font écho à et parfois même anticipent une réflexion philosophique ou poétique. Le grand tournant d'idées qui s'effectue à l'époque se voit ainsi accordé une envergure encore plus large. Il la mérite encore parce que le patrimoine culinaire de la France, c'est à dire un ensemble où savoir-manger et apprêts se confondent, s'enrichit au dix-huitième siècle de façon exponentielle. Il s'agit, soulignons-le, d' un dix-huitième siècle 'long,' à savoir, celui qui empiète aux deux bouts sur les siècles attenants. Enfin, et comme cela se dit si bien en anglais, last but by no means least, il la mérite parce qu'un tel discours situe les fondements d'une mentalité et d'une image de marque bien françaises d'aujourd'hui. Sans avoir à évoquer les divers guides gastronomiques dont les classements font loi dans le monde entier, il faut noter que les sites internet prennent la relève. Il en existe pour le fromage (www.fromages.com) comme pour le foie gras (www.newtechr.fr/rayssaguel/), sans parler des vins. ${ }^{1}$ Dans sa Physiologie du goût, qui date de 1826, Brillat-Savarin note d'ailleurs que le mot 'gourmandise' est intraduisible, et il clot son ouvrage par un banquet voué au culte de Gastéréa, une muse de son invention, dont le temple se situe, cela va de soi, à Paris.

Le culte de Gastéréa - inspiratrice des plaisirs gustatifs - est issu d'une tradition littéraire française qui remonte (au moins) jusqu'à la Renaissance. Il s'exprime à travers des poèmes dits gourmands, et de manière hyperbolique dans la prose de Rabelais. ${ }^{2}$ Messire Gaster et les gastrolâtres (dont on retrouve d'ailleurs la trace dans le Neveu de Rameau de Diderot) participent cependant d'un monde où ce qui tient des plaisirs du palais et des moyens d'y pourvoir ou d'y accéder reste relégué dans le 'bas,' et se trouve parsuite exilé du 'noble.' C'est durant le siècle des Lumières que s'opère la consécration du fait culinaire et que surgissent les croyances, doctrines et rituels qui l'anoblissent, qui le font passer du gaulois au savant, qui transforment le glouton en gourmet. 
Le phénomène n'est toutefois pas purement français. Des aliments venus du Nouveau Monde - ils sont nombreux - viennent petit à petit s'implanter en Europe et font redessiner la carte des mets: dinde, pomme de terre, maïs, tomate, arachide, cacao, maintes courges et fèves, pour ne citer que les plus marquants. ${ }^{3}$ Il existe dans l'espace culinaire, comme ailleurs, des échanges, des recoupements, plus particulièrement en ce qui concerne la France et l'Angleterre. Ceci dit, on peut trouver une ébauche du Guide Michelin dans l'Almanach des gourmands de Grimod de la Reynière (années Consulat-Empire), donc en France. Les grands chefs qui se singularisent dès la fin du dix-septième siècle et sont convoités à l'étranger sont tous français. Lorsque Diderot, dans une lettre du 9 septembre 1767, parle en termes louangeux du restaurateur de la rue des Poulies, il évoque ce qu'il est convenu d'appeler le premier restaurant. Les sauces liées sont bien des créations des Lumières françaises: il n'y a qu'à parcourir les listes qui n'en finissent pas dans certains livres de recettes, par exemple Les Soupers de la cour (1755), pour s'en convaincre. Quant au champagne, il est 'inventé' par Dom Pérignon vers 1668, puis chanté dans Le Mondain de Voltaire un peu plus d'un demi-siècle plus tard, et s'il existe du foie gras dès l'époque romaine et sans doute avant, sa mise en pâté date du dix-huitième siècle. La pâtisserie monumentale - véritable architecture du sucré - voit également le jour au dix-huitième siècle. Le grand Antonin Carême, maître d'hôtel de Talleyrand et de têtes couronnées, $s^{\prime}$ illustrera dans cette voie, à la lettre celle de la haute cuisine. Cette dernière est à ne pas confondre avec la gastronomie, qui se manifeste lexicalement comme autrement tout au début du dix-neuvième siècle. ${ }^{4}$ Bien plus proche du gastrosophe de Charles Fourier que du gastrolâtre de Rabelais, le gastronome sait manger. Sans connaisseurs, pas de fine cuisine. Il y aurait bien d'autres exemples à fournir pour accuser le caractère quintessentiellement français de cette émergence du culinaire.

Revenons au titre. Quels sont, au dix-huitième siècle, les liens entre savoirs et saveurs? Dire que le savoir c'est à l'époque le désir d'en savoir plus, mieux, et autrement serait un lieu commun. Il n'est donc pas étonnant qu'au niveau de l'imprimé les recueils de savoir de toute espèce, dictionnaires, almanachs, manuels, traités, etc., avec, bien entendu, l'Encyclopédie en tête, soient en bonne place, tout comme l'effort d'étendre la diffusion de ce savoir par la presse et les livres de colportage, entre autres moyens. C'est grâce à ceux-ci que les saveurs se sauront, que le savoir-goûter ira à la rencontre d'un savoir-manger nouveau, comme d'un savoir-vivre et d'un savoir-faire. Les mots 'saveur' et 'savourer,' si l'on s'en tient au seul investissement de l'Encyclopédie, paraissent respectivement sous la plume de Jaucourt et de Diderot. ${ }^{5} \mathrm{D}^{\prime}$ après Diderot, on savoure la douceur d'un mets comme on savoure le plaisir (vol.14, 
p.723). Cette intrication du littéral et du figuré se retrouve dans le cas d'un parent proche, le mot 'goût. ${ }^{6}$ On peut trouver d'autres exemples, comme l'article 'délicieux' ou 'faim, appétit,' tous deux de Diderot. Cela revient à accorder à ces termes une dimension abstraite, annoblie si l'on veut, voire artiste. Rappelons que le mot 'esthétique' date du dix-huitième siècle et qu'il renvoie à un mot grec, aisthanesthai, qui signifie sentir. A rappeler également: le gustatif, ainsi que les autres sens qui font partie du culinaire - c'est-à-dire tous - occupent une place centrale dans toute philosophie sensualiste ainsi que dans toute celle qui se veut expérimentale et qui a ses assises dans le monde de la science et, par extension, dans celui de la technologie. Enfin, comme pour toute question de saveur les goûts diffèrent (ce qui est bon selon les uns ne l'est pas nécessairement selon d'autres), on voit se former un discours moral sur le pur et l'impur, le naturel et le culturel, le sain et le malsain en matière de ce qui relève des saveurs et du cuisiné. Telles sont, en somme les grandes facettes du sujet: le scriptural, le philosophique, l'artiste, le scientifique, et le moral. Dans ce qui suit, il ne s'agira guère de recettes ou de plats spécifiques, si innovateurs soient-ils, ni de grands festins ou de petits soupers. ${ }^{7} L^{\prime}$ accent sera mis sur l'entrée des saveurs dans le monde du savoir et les moyens par lesquels elle s'effectue.

Le fait capital, le point de convergence du grand tournant est la mise en écriture du culinaire. Selon Alain Girard, le nombre de livres de cuisine publiés avant 1650 ainsi que l'importance de leurs tirages sont négligeables, là où le siècle et demi à suivre accuse une montée exponentielle de ces livres, qui deviennent par conséquent un phénomène de marché dont il faut tenir compte. Cette évolution s'explique sans doute par celle du marché du livre en général mais tient certainement aussi à $\mathrm{d}^{\prime}$ autres facteurs, dont le moindre n'est pas celui des cuisiniers hauts de gamme - maîtres queux, maîtres d'hôtel, officiers de bouche, chefs de cuisine - qui se mettent à écrire. Parmi les plus en vue de l'époque en question on trouve François Pierre (dit La Varenne), Pierre de Lune, Massialot (auquel renvoie Voltaire à plus d'une reprise), Vincent La Chapelle, et François Marin. Ajoutons que s'il existe une connivence entre aliments et santé depuis le temps des Grecs, ce n'est qu'à l'époque qui nous intéresse que des médecins dont la réputation va au-delà des frontières se mettent à écrire des traités culinaires bona fide, l'exemple par excellence étant celui de Louis Lémery et son Traité des aliments, d'abord publié en 1702, et ceci à un moment où les sciences de la nutrition et la notion de santé publique émergent petit à petit. ${ }^{8}$ Enfin, $1^{\prime}$ approvisionnement en denrées alimentaires posant moins problème, le critère du bon repas passe du quantitatif au qualitatif.

Ce qu'il convient d'appeler, faute d'un terme plus précis, les écrits culinaires du dix-huitième siècle, se répartissent grosso modo en quatre 
catégories: les manuels de cuisine à proprement parler, les écrits discursifs tels que préfaces ou courts pamphlets, certains articles de l'incontournable Encyclopédie, et ce qui se dit parfois 'écriture gourmande,' en l'occurrence, des textes de Grimod de la Reynière et de Brillat-Savarin. La fiction narrative, bien que riche en possibilités de lecture sitiocritique, n'entrera pas dans le présent propos. ${ }^{9}$ Il sera surtout question des deux premières catégories citées ci-dessus.

Les livres de cuisine du dix-huitième siècle diffèrent de ceux de nos jours à bien des égards. Aujourd'hui, on verse dans les modes, celles du terroir ou de l'ethnique par exemple, ou celles qui s'associent à une croyance ou à un engagement (p.e. la cuisine végétarienne ou la cuisine cacher). Ces postulations culinaires sont absentes au dix-huitième siècle au niveau de la recette imprimée, du moins en France. Ce que l'on voit par contre, ce sont des titres qui soulignent une stratégie de diffusion non seulement livresque mais aussi sociale. L'éventail s'étend depuis $L e$ Cuisinier royal et bourgeois de Massialot, dont la première édition date de 1691, au Petit cuisinier économe (ouvrage anonyme datant de 1796), en passant par La Cuisine des pauvres, attribué à Varenne de Béost (1772) et la menue (moins de quarante pages) mais néanmoins robuste Cuisinière républicaine de Mme Mérigot parue en l'an III, sans négliger le best-seller du siècle en son genre, La Cuisinière bourgeoise de Menon, dont la première d'un très grand nombre d'éditions date de 1746.

La tendance est nette: on vise des échelons sociaux spécifiques. Il en résulte une ouverture de plus en plus marquée du savoir culinaire. On trouve par-ci des recettes de plusieurs pages qui nécessitent un personnel de cuisine important et plusieurs jours pour les mener à bien; par-là, quelques unes de trois à quatre lignes dont l'unique ingrédient est la pomme de terre. ${ }^{10} \mathrm{Vu}$ par le biais des livres de recettes, il y a un embourgeoisement sinon une démocratisation du culinaire. Chose curieuse pourtant: l'utilisateur/trice d'un livre de cuisine doit naviguer sans balises. Pas de temps de cuisson, encore moins d'indication de température; la précision des quantités est assez rare, celle du poids quasi inexistante. On suppose donc une connaissance pratique au préalable et, bien entendu, un utilisateur qui sache lire. Il en va de même pour les illustrations. Pas de méthode Pépin qui vous montre comment tenir la cuiller en remuant votre purée. ${ }^{11} \mathrm{~L}$ 'iconographie culinaire du dix-huitième siècle a une allure autre et un autre but. Il n'y est point question, comme dans les livres de cuisine d'aujourd'hui, de faire saliver le lecteur qui contemple la photo en couleurs d'un plat dans toute sa splendeur saucée ou croustillante, mais de rehausser la dimension discursive du contenu au moyen de frontispices allégoriques, tel celui des Dons de Comus, ou fantaisistes (tels ceux des volumes de l'Almanach des gourmands). D'autres types d'illustrations (objets décoratifs, plans de table, 
etc.) soulignent que certains manuels sont à l'usage d'intendants, c'est à dire de chefs qui orchestrent de grands repas.

La recette de type bourgeois est particulièrement prônée par Diderot, vu celles qu'il contribue à l'Encyclopédie: boeuf à la mode, biscuit, compote d'abricots, etc.... L'appellation 'à la mode' fait penser par ailleurs à la classe moyenne montante, dont les hôtes, à l'instar de Monsieur Jourdain, veulent faire les gentilshommes. C'est dire qu'un mets 'bourgeois' n'est pas nécessairement simple. Dans ses Dons de Comus, François Marin se moque des repas ostentatoires de ces nouveaux riches.

Il y a des manuels qui s'en tiennent au simple et d'autres au recherché. Certains cumulent les deux, comme Le Cuisinier moderne de Vincent La Chapelle. ${ }^{12} \mathrm{~L}^{\prime}$ auteur y est tiraillé entre le désir de secourir culinairement les défavorisés (il y a, par exemple, toute une section consacrée aux recettes à l'intention de gens faibles et malades) et le fait qu'en tant que chef de cuisine d'abord de Lord Chesterfield et ensuite de Guillaume d'Orange il est chargé de préparer des repas d'apparat. Ce qui, mis à part le titre d'un manuel, laisse entrevoir le type de lecteur envisagé n'est peut-être pas tant la nature du contenu que sa présentation. Le Cuisinier français de La Varenne, dont l'une des nombreuses rééditions fut colportée, vient ici en tête de file. ${ }^{13}$ Les recettes y sont souvent modulaires, c'est à dire à parties interchangeables ou à variantes en 'autre façon.' On peut trouver la même souplesse substitutive ailleurs. Cette approche au culinaire est nouvelle, tout comme, dans ce même livre de cuisine, la sauce à base de roux, une invention qui selon certains serait aussi importante que celle de la roue.... Ce qui est nouveau ou inventif se faufile inévitablement dans d'autres manuels. A une époque où on ne connaît pas encore les droits d'auteur, il y a des glissements de recettes un peu partout, ce qui mène parfois à des guerres larvées bien comiques. Exemple savoureux d'espionnage à l'échelle des rots, des pâtés, et des sauces!

Passons aux écrits discursifs, qui s'intègrent d'ailleurs souvent dans des manuels, dans la mesure où ils en constituent les préfaces ou les avertissements. Une fois de plus, on se trouve devant une divergence sensible entre les livres de cuisine d'aujourd'hui et ceux des Lumières. Julia Child ou Paul Bocuse versent dans le médiatique bien plus que dans l'idéologique et une philosophie du plat ne leur viendrait guère à l'idée. Par contre, nombreux sont les manuels du dix-huitième siècle qui se placent dans l'un ou plusieurs de trois grands champs discursifs: l'historique, le moral, l'artiste. On pourrait même dire que les titres de manuels, qui remplissent à l'occasion une page entière, sont des minidiscours lorsqu'ils profilent le destinataire idéal du livre et précisent comment il faut s'y prendre pour s'assurer du bon usage du livre en question. 
Du côté de l'histoire, malgré certaines voix discordantes, on se veut nettement progressiste. Il n'y a pour ainsi dire nul préfacier qui ne trouve le moderne supérieur à l'ancien. Prenons comme exemple le Dictionnaire des aliments (1750; attibué à Briand) qui spécifie sur sa page de titre qu'il s'agit de 'la manière ancienne et moderne' d'apprêter les aliments. Dans sa préface, l'auteur se situe dans la mouvance des jésuites Brumoy et Bougeant, à qui l'on doit vraisemblablement le long - 35 pages 'Avertissement' des Dons de Comus, et qui déclarent que 'la cuisine, comme tous les autres arts, s'est perfectionnée avec le génie des peuples, et elle est devenue plus délicate à mesure qu'ils se sont polis' (p.iii). Une affirmation semblable se trouve dans un Traité historique et pratique de la cuisine de Menon (1758), chez le janséniste Foncemagne dans une importante 'Dissertation préliminaire' de La Science du maître d'hôtel cuisinier de Menon (1749), et s'exprime en termes dithyrambiques sous la plume de Grimod de la Reynière dans la préface de son Manuel des amphitryons (1808). Ce perfectibilisme culinaire s'étend d'ailleurs bien au-delà des marmites et des marmitons: 'Sera-ce donc trop s'avancer,' déclare Foncemagne, 'de placer les apprêts de la cuisine moderne parmi les causes physiques qui du sein de la Barbarie ont rappelé parmi nous le règne de la politesse, des talents de l'esprit, des arts et des sciences?' (p.xix). La civilisation entière y passe.

Tournons-nous maintenant du côté de l'Encyclopédie, qui contient à l'article 'cuisine,' signé Jaucourt, un point de vue plus mitigé. Sans se départir d'un engagement moderniste, il lance l'alarme: le luxe de la bonne chère peut tourner en luxure et peut 'défigurer de cent manières différentes les mets que donne la nature,' menant ainsi, conclut l'auteur, à un lamentable dérèglement du goût' (vol.4, pp.537-39). Ici surgit l'antique discours sur le luxe, qui condamne les excès de tous genres et met en avant le simple. Le simple... et le naturel. Si les Modernes valent mieux que les Anciens, ceux-ci, dits 'nos ancêtres,' s'entourent d'une nimbe ornée d'une nostalgie d'âge d'or. En fait, la nature avait déjà lancé son défi à la culture quelques années auparavant, avant même le Discours sur les sciences et les arts de Rousseau. Un dénommé Desalleurs satirise en 1739 le perfectibilisme des préfaciers jésuites dans leur Avertissement des Dons de Comus et se moque de tout ce qui s'éloigne 'de la rustique simplicité de nos pères' tout comme de plats qualifiés de 'géométriquement chimiques' (pp.4 et 11). D'autres se mêleront par la suite de ce qui tournera en une polémique opposant sybarites et naturistes, finement analysée en 1981 par Stephen Mennell. A ce titre, il faut mentionner Meusnier de Querlon, qui se positionne du côté de la culture dans une 'Apologie des modernes' qui lui est attribuée et qui servira ensuite de préface à une Suite des Dons de Comus (1742). Comme d'autres plumitifs préfaciers, il varie ses modes d'écriture et on lui doit, entre autres 
ouvrages, des textes bien libertins tel que l'Histoire galante de la tourière des Carmélites (1743). Quant aux pères Brumoy et Bougeant, ils ne restent pas toujours dans la droite ligne des enseignements de Trévoux, puisqu'ils font remonter le goût corporel au goût spirituel. Enfin, aux côtés de ces discours puristes et naturistes on trouve celui de la diététique. Le Dictionnaire des aliments vise à rendre la nourriture 'salutaire.' Il y a de nombreux échos, dont le plus résonnant est celui de Jourdan Le Cointe dans sa Cuisine de santé (1790). Autrement dit, les saveurs se doivent d'être salubres.

Ce qu'il y a sans doute de plus original et peut-être de plus significatif dans ces déclarations liminaires, c'est leur poétisation, à savoir la transposition dans un espace artiste du monde du culinaire. Pour Brumoy et Bougeant, le chef est un artiste créateur à qui l'on doit 'cette Union que les Peintres donnent aux couleurs,' à laquelle s'ajoute 'une harmonie de tous les goûts ensemble' (p.xx). Foncemagne pousse la synesthésie encore plus loin en constatant 'qu'il y a l'harmonie des saveurs, comme l'harmonie des sons, et peut-être celle des couleurs et des odeurs' (p.viii). Nous ne sommes pas loin de Baudelaire. Dans le sillage d'une approche sensualiste à la notion du beau (comme, par exemple celle de Du Bos), la cuisine - du moins la haute - est agréée l'un des beaux arts à part entière. Il y aura même, grâce à l'inspiration du récollet Polycarpe Poncelet, un rendu sonore des saveurs au moyen des 'vibrations' que celles-ci produisent, sous forme d'une gamme de sept saveurs dont chacune correspond à une note, et dont la partition se trouve dans la Chimie du goût et de l'odorat (1755)!

De nos jours, on trouve parfois des auteurs culinaires inattendus comme l'ex-chancelier Kohl. Vu la pléthore de livres de cuisine dont le succès en librairie dépend souvent de la célébrité d'un personnage médiatisé ou de belles photos en couleur de plats qui font rêver ou baver (prises par des spécialistes en la matière dits 'food artists' aux EtatsUnis), l'auteur d'un livre de cuisine ne passe pas inaperçu. Et les femmes tiennent bien la tête aux hommes. Aux dix-huitième siècle, il est loin d'en être de même. ${ }^{14}$ Les chefs scripteurs doivent leur réputation bien plus à leur talent devant les fourneaux qu'à leurs écrits, et le nombre de manuels paraissant sous le signe de l'anonymat est loin d'être négligeable. On s'intéresse relativement peu à leurs auteurs, et certains d'entre eux restent entourés de mystère. A titre d'exemple: Menon, l'auteur culinaire français le plus prolifique du siècle. Il s'agit en toute vraisemblance d'un pseudonyme. La biographie Michaud retrace les étapes de sa production livresque mais avoue ignorer l'identité du personnage. Parmi les auteurs de manuels, il y en a deux qui intriguent tout particulièrement. Tout d'abord, Vincent La Chapelle, premier chef auteur à s'expatrier. Il s'agit vraisemblablement d'un huguenot exilé, ou fils 
d'exilé, qui vit d'abord à Londres, où il est membre d'une loge maçonnique dite French Union Lodge. Passant ensuite du service de Lord Chesterfield à celui de Guillaume d'Orange, il s'établit à La Haye, où paraît en 1735 une splendide édition de son Cuisinier moderne, et ceci à frais d'auteur. Il devait donc être bien rémunéré. Selon certains indices, il aurait été le premier maître de la première loge maçonnique hollandaise. Ses recettes se remarquent par un authentique fumet d'Orient. Peut-être avait-il des attaches à la Compagnie des Indes Orientales, vu les 'instructions pour un cuisinier ... qui s'embarque pour la marine' que contient son livre et qui en occupent toute une section. L'autre auteur hors série - il ne s'agit que d'une attribution, mais elle est bien fondée - est celui à qui l'on doit Le Cuisinier gascon, qui n'a d'ailleurs strictement rien de gascon ou de régional, mais tient plutôt de la gasconnade. Tout porte à croire que l'auteur n'est autre que le prince de Dombes, petit-fils de Louis XIV et parsuite un prince du sang. Chose bien curieuse pour l'époque.

Il faut toutefois attendre la décennie révolutionnaire pour trouver un livre de cuisine rédigé par une femme. A notre connaissance, il n'y en a qu'un seul en France durant tout le siècle: La Cuisinière républicaine de Mme Mérigot (an III). Encore a-t-on affaire à une veuve de libraire, et à un livre consacré uniquement à la pomme-de-terre. Les choses se passent pourtant bien différemment en Angleterre, où les auteurs femme vont de l'avant. Le best-seller outre-Manche est d'une femme, The Art of Cookery Made Plain and Easy (de Hannah Glasse, 1747). Une autre qui ne passe pas inaperçue se nomme Elizabeth Raffald, auteur, inter alia, de l'Experienced English Housekeeper (1769), mère, à ce qu'on dit, de seize enfants, toutes des filles, et qui trouvait encore le temps de s'occuper d'un restaurant.

Malgré un parti pris moderniste généralisé, un nombre non négligeable des manuels du dix-huitième siècle s'en tiennent encore à la médecine hippocratique des humeurs et recommandent tel ou tel aliment en fonction du tempérament du mangeur. Tel est le cas du Traité des aliments de Louis Lémery, ainsi que du Dictionnaire des aliments, où l'on trouve, par exemple, à propos des citrouilles qu'elles 'conviennent dans les temps chauds aux jeunes gens bilieux, mais les vieillards et ceux qui sont d'un tempérament faible et flegmatique doivent s'en abstenir' (vol.1, p.392). L'investissement diététique de la médecine des humeurs d'une part, le discours sur la salubrité de l'autre, se répercutent sur les savoirs-saveurs. 'Quel est dans le fond l'objet du cuisinier si ce n'est ... d'aider les fonctions de l'estomac en excitant ses facultés?' lit-on dans 1'Avertissement des Dons de Comus (pp. xxiii-iv).

A côté de recettes destinées à la table des grands, on trouve dans le Cuisinier moderne une section consacrée aux recettes pour malades et 
vieillards, une autre à celles conçues pour les voyages maritimes au long cours. Cette dernière se concentre sur le problème capital de la conservation des vivres, solutionnée (ou pas) de diverses manières. On y trouve par exemple un ancêtre du bouillon cube. Je rappelle qu'Appert, à qui l'on doit l'invention de la boîte à conserve, perce, si l'on peut dire, à la fin du siècle. La science des sucs est bien entendue également véhiculée par l'Encyclopédie. A côté d'articles se rangeant dans le champ du gustatif (assaisonnement, cuisson, délicieux, saveur, etc.) on trouve ceux qui entrent dans le physiologique - digestion, régime, tempérament - où l'apport des médecins est à signaler, avant tout celui de Venel et de d'Aumont. Hippocrate et Galien sont des références obligées, le nom de Boerhaave paraît de temps à autre, Bordeu est cité à l'occasion, comme dans l'article 'digestion,' qui est de Venel. On trouve toutefois dans 'tempérament' (un article non signé) la constatation que 'dans la médecine d'aujourd'hui on considère beaucoup moins les tempéraments' (vol.16, p.6). Le régime alimentaire, afin de maintenir un équilibre des humeurs, doit tenir compte des affinités existant entre tempéraments et aliments.

Certains des articles mentionnés ci-dessus sont de Diderot. L'article 'avaler' est également de lui. Son contenu pittoresque mérite d'être cité, ne serait-ce que de façon abrégée. Voici donc des fragments de la partie centrale: 'Une fille âgée de dix ans ... avala en jouant un couteau de six pouces et demi ... le couteau changea de place plusieurs fois, et cessa $\mathrm{d}$ 'incommoder cette fille au bout de quelques mois: mais un an après on ne le sentit presque plus, tant il avait diminué: enfin il sortit par un abcès que sa pointe avait causée trois travers de doigt au-dessus du creux de l'estomac; mais il était extrêmement diminué, et la fille fut entièrement rétablie' (vol.1, p.858). Côté médecine, le savoir-saveur bascule nettement entre la science et le folklore!

En conclusion, comment envisager l'ensemble de tous ces phénomènes? On est tenté d'établir un parallèle entre leurs cheminements, du moins au niveau de l'écrit, et celui du roman. Dans les deux cas on peut observer l'engrenage d'un avènement et d'un tournant. L'avènement est celui de la haute cuisine et de l'intégration du culinaire dans le monde de la civilité. Le tournant est celui qui ouvre la voie aux grands épanouissements qui suivront, aux chefs qui trôneront au Savoy de Londres tel Escoffier, au mangeur du dix-neuvième siècle tel que nous le présente Jean-Paul Aron, aux repas d'apparat officiels de la Troisième République et d'aujourd'hui tels qu'en parle Pascal Ory. Mais ce même tournant mène aussi vers la nouvelle cuisine, la cuisine minceur, et celle à base de produits bio-naturels. Un article intitulé 'Mieux manger pour mieux vivre' ayant paru en août 1998 dans le Nouvel Observateur souligne 
la valeur nutritive et les effets salutaires du foie gras. Gastéréa, la muse de Brillat-Savarin, reste fidèle au poste et veille à ses adeptes.

\author{
BEATRICE FINK \\ University of Maryland
}

\title{
Notes
}

1 Voir le Washington Post du 22.3.98.

2 Sans oublier le passage que Montaigne consacre à la 'science de gueule' dans ses Essais (Paris: Garnier, 1962), vol. I, p.339.

3 Parmi les nouvelles denrées alimentaires, deux se distinguent par leur importance et le fait qu'elles ne sont pas originaires d'Amérique: le thé (Asie) et le café (Proche Orient). Certains des aliments nouveaux (p.e. la pomme de terre et la tomate) ont de la peine à s'implanter en Europe et plus particulièrement en France parce qu'on les croit porteuses de maladies. Notons également un certain flou lexical: le maïs s'appelle d'abord 'blé de Turquie,' la pomme de terre 'truffe' ou 'topinambour,' de même que la tomate est servie lexicalement à diverses sauces.

4 Voir le poème didactique en quatre chants de Joseph Berchoux intitulé $L a$ Gastronomie, ou l'homme des champs à table (1801) qui fait entrer le mot dans le lexique français.

5 Dans le cas de Diderot il s'agit, selon Jacques Proust, d'une attribution possible.

6 Il existe deux entrées distinctes dans l'Encyclopédie pour le mot 'goût': la première (à laquelle nous renvoyons) est de Jaucourt et se classe sous 'physiologie.' La deuxième, bien plus longue, se classe sous 'grammaire, littérature, philosophie.' Elle est signée Voltaire et contient en appendice un passage de Montesquieu sur le goût.

7 Les gourmands pourront se consoler de cette absence en consultant l'appendice.

8 Voir ci-dessous dans notre texte les articles 'culinaires' de l'Encyclopédie qui sont signés par des médecins.

9 Ce mot 'sitiocritique' désigne une approche critique qui cible le signe alimentaire, culinaire, ou prandial.

10 Bien qu'Olivier de Serres en parle dès son Théâtre d'agriculture et ménage des champs (1600) et qu'on mange de ces 'racines' dans le nord-est de la France à l'aube du dix-huitième siècle, la pomme de terre devra attendre Parmentier et l'époque révolutionnaire pour qu'elle ait droit à sa place au soleil.

11 Jacques Pépin est un chef et auteur culinaire vivant aux Etats-Unis dont la très didactique 'méthode' a connu une grande vogue outre-Atlantique durant les années 80 .

12 D'abord publié en anglais (Londres, 1733) sous le titre The Modern Cook, puis en français à La Haye en 1735. Dans les deux cas, ce fut à frais d'auteur. 
13 L'édition colportée fut récemment rééditée (Paris: Montalba, 1983) et enrichie d'une longue introduction de Jean-Louis Flandrin et de Philip et Mary Hyman.

14 Sauf en Angleterre, comme on le verra ci-dessous.

\section{Liste des ouvrages cités ou consultés}

1. Anonyme. Le Cuisinier gascon. Amsterdam, 1740.

2. Le Letit cuisinier econome. Paris: Janet, 1796.

3. Aron, Jean-Paul, Le Mangeur du XIXe siècle. Paris: Laffont, 1973.

4. Biographie universelle Michaud. 2ème édition, vol.XXVII.

5. Briand (attr.). Dictionnaire des aliments. Paris: Gissey, 1750.

6. Brillat-Savarin, Jean-Anthelme. Physiologie du goût. Paris: de Bonnot, 1986 (1ère édition: 1826).

7. Dix-huitième siècle, no 15 (1983), Aliments et cuisine.

8. Du Bos. Réflexions critiques sur la poésie et sur la peinture. Paris: 1770 (1ère édition: 1719).

9. Encyclopédie, ou dictionnaire raisonné des sciences, des arts, et des métiers. Paris: Briasson et al.,1751-65.

10. Fink, Béatrice. Les Liaisons savoureuses. Saint-Etienne: Publications de l'Université de Saint-Etienne, 1995.

11. Girard, Alain. 'Le Triomphe de la cuisinière bourgeoise,' Revue d'histoire moderne et contemporaine, vol.XXIV, pp.497-523.

12. Glasse, Hannah. The Art of Cookery Made Plain and Easy. Londres, 1747.

13. Grimod de la Reynière, A.B.L. Almanach des gourmands. Paris: Maradan, 1803-12.

14. . Manuel des amphitryons. Paris: Métailié, 1983 (1ère édition: 1808).

15. L.S.R. L'Art de bien traiter. Paris: Du Puis, 1674.

16. La Chapelle, Vincent. Le Cuisinier moderne. La Haye: De Groot, 1735 (1ère édition: 1733, en anglais).

17. La Varenne. Le Cuisinier français. Paris: Montalba, 1983 (1ère édition: 1651).

18. Le Cointe, Jourdan. La Cuisine de santé. Paris: Briand, 1790.

19. Lemery, Louis. Traité des aliments. Paris: Witte, 1705 (1ère édition: 1702).

20. Marin, François. Les Dons de Comus. Paris: Prault, 1739. Préface attribuée à Brumoy et Bougeant.

21. _. Suite des Dons de Comus. Paris: Pissot, 1742. Préface attribuée à Meusnier de Querlon.

22. Massialot. Le Cuisinier royal et bourgeois. Paris: De Sercy, 1691. 
23. Mennell, Stephen. Lettre d'un pâtissier anglais et autres contributions à une polémique gastronomique du XVIIIe siècle. Exeter: Exeter University, 1981.

24. . All Manners of Food.. Oxford: Blackwell's, 1985.

25. Menon. La Cuisinière bourgeoise. Bruxelles: Foppens, 1774 (1ère édition: 1746).

26. _. La Science du maître d'hôtel cuisinier. Paris: Libraires associés, 1789 (1ère édition: 1749).

27. __ Les Soupers de la cour. Paris: Guillyn, 1755.

28. . Traité historique et pratique de la cuisine. Paris: Bauche, 1758.

29. Mérigot, Mme. La Cuisinière républicaine. Paris: Mérigot jeune, an III.

30. Le Nouvel Observateur. $\mathrm{N}^{\mathrm{o}} 1761$ (6-12 août 1998).

31. Ory, Pascal. Le Discours gastronomique français. Paris: Gallimard, 1998.

32. Poncelet, Polycarpe. Chimie du goût et de l'odorat. Paris: Le Mercier, 1755.

33. Serres, Olivier de. Le Théâtre d'agriculture et ménage des champs. Paris, Métayer, 1600.

34. Varenne de Beost (attr.). La Cuisine des pauvres. Dijon, 1772.

\section{Appendice}

La présentation orale du texte ci-dessus fut précédé d'un repas festif dont le menu consistait uniquement en plats à base de préparations datant du dix-huitième siècle d'origine canadienne ou française. Ce repas fut réalisé sous la haute surveillance de Kathryn Merrett. Nous reproduisons ici un abrégé des notes explicatives, rédigées par la même.

\section{Menu du banquet}

Le premier plat est une polenta, préparation culinaire épaisse à base de maïs et de champignons. C'est un plat $d$ 'inspiration nord-américaine. Les tribus iroquoises, qui habitaient les régions autour de Grands Lacs, cultivaient plus de quinze variétés de maïs. Ils consommaient aussi des haricots et des courges supplées par du poisson, du gibier, des noix et des racines. Les coureurs des bois et les immigrants trouvèrent que le maïs était plus facile à moudre que le blé. Ils le préparaient en une espèce de bouillie - mélangée avec des courges - coupée en tranches et nappée de sucre d'érable ou frite. 
Le plat principal consiste en une préparation de saumon aux fines herbes dont la recette provient des Soupers de la cour. Les darnes de saumon - panées de chapelure fine, enduites de beurre fondu auquel on ajoute du persil, des ciboules [scallions], des échalotes, une pointe d'ail, des champignons, un peu de basilic (le tout haché très fin), sel et gros poivre - sont gratinées au four. Le tout était autrefois accompagné d'une sauce 'italienne' à base de champagne, de consommé, d'un peu de coulis, de champignons et d'une tranche de jambon (version simplifiée servie au repas d'Edmonton). Ce plat est accompagné de petits pois à la bourgeoise dont la recette est tirée de la Cuisinière bourgeoise et de pommes de terre préparées selon une recette très simple venant de $L a$ cuisinière républicaine.

Pour le dessert, retour aux denrées nord-américaines du dix-huitième siècle. Le riz sauvage (en fait, une variété d'avoine), était cueilli et consommé par les aborigènes et connu des marchands et des immigrants. Le dictionnaire Gage des canadianismes cite des références remontant jusqu'à 1744 . Le sirop d'érable (la sève de l'arbre) était vénéré par les aborigènes de Woodland, qui voyaient en cet arbre un don du Créateur, et la coulée de la sève comme le premier signe du printemps. On ne peut pas remonter aux origines de la recette de tourtes de riz sauvage et de sucre d'érable. Nous remercions toutefois le Créateur de nous en avoir fait don.

$* * * * *$

Le repas fut suivi d'une séance de dégustation de trois vins doux, ce type de vin étant très prisé au dix-huitième siècle: un porto Dow, un vin de Madeire Cossard et Gordon, et un Jurançon moelleux. John Rempel fut le maître d'oeuvre de cette séance. 
52 Beatrice Fink

\section{Illustrations}

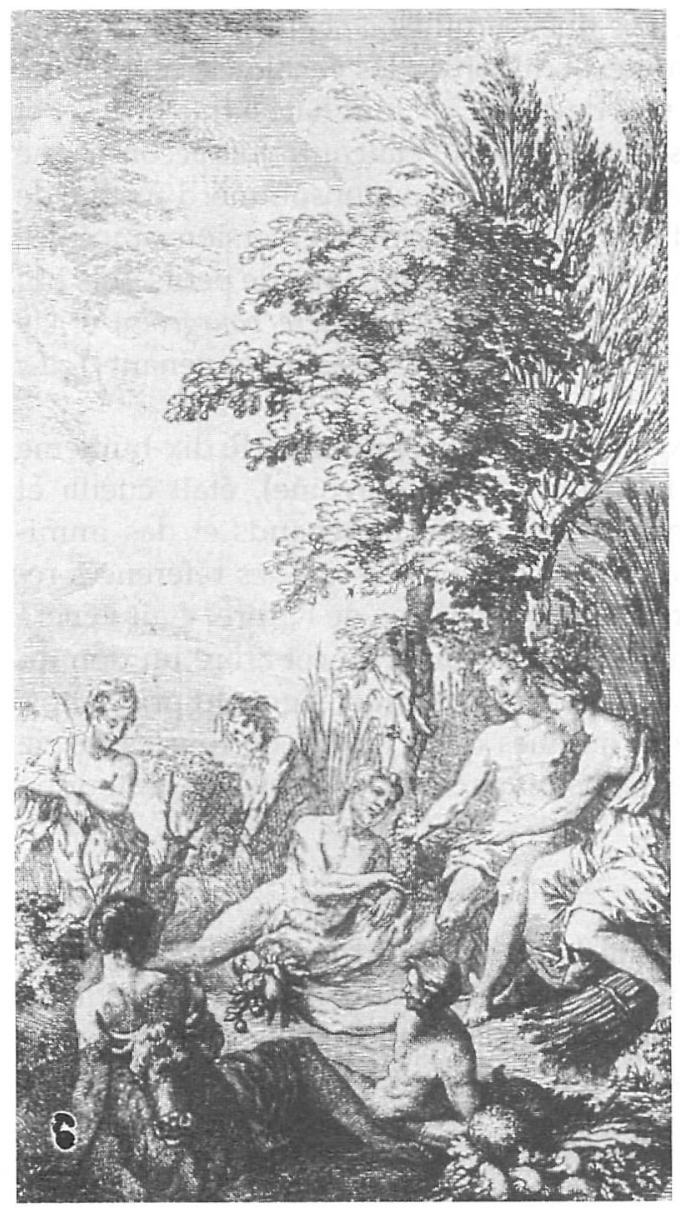

\section{Figure 1}

Les Dons de Comus.

Frontispice. 


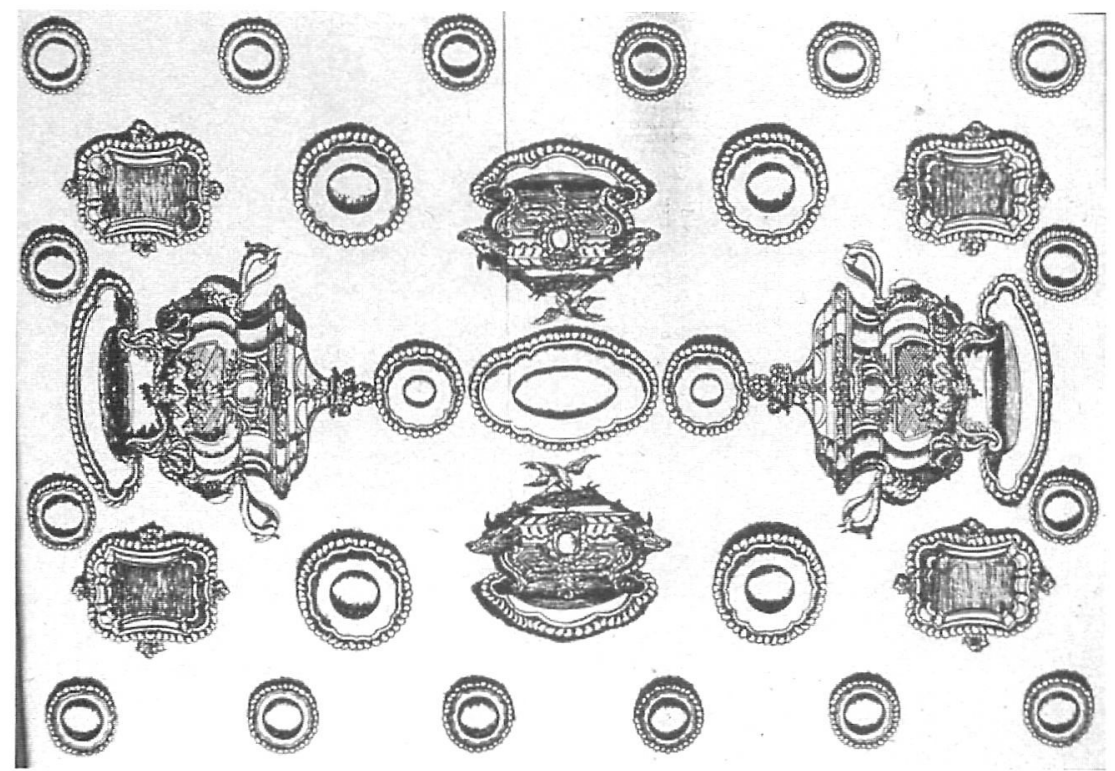

Figure 2 Le Cuisinier Moderne. Ornements de table.

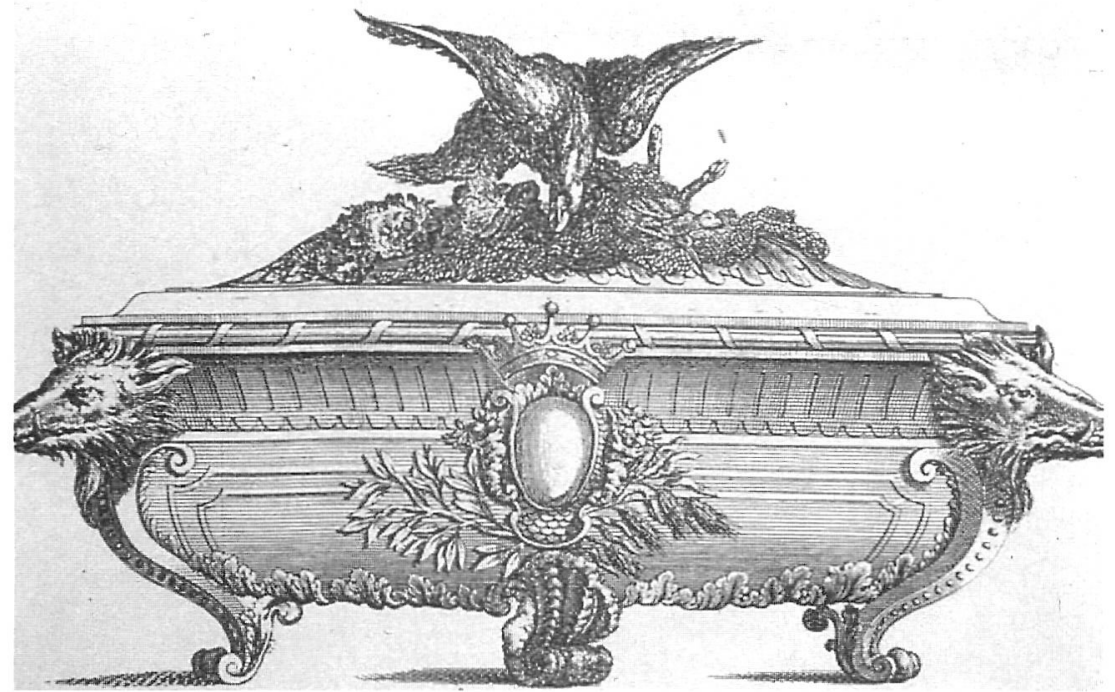

Figure 3 Le Cuisinier Moderne. Terrine décorative. 